\title{
Análisis del impacto de la violencia en la situación económica y el ejercicio de la libertad de expresión en un grupo de periodistas veracruzanos
}

\author{
Violeta Santiago ${ }^{1}$
}

\begin{abstract}
Resumen
Esta investigación cuantitativa tiene por objetivo describir el panorama laboral, económico, de seguridad y libertad en el que un grupo de periodistas desarrollan su trabajo en el marco de una situación de alta violencia en el estado de Veracruz, el más peligroso para ejercer esta profesión en México. Adicionalmente, el trabajo propone la existencia de una correlación entre el hecho de sufrir violencia y la construcción de su percepción, así como entre violencia y situación económica y violencia y libertad de expresión. Los resultados de este estudio demuestran que la idea de violencia en el entorno de los periodistas analizados está relacionada con el grado de exposición directa a la violencia (ataques, amenazas, agresiones) a las que se han expuesto, además de que existe una correlación positiva entre la exposición a la violencia (construida por la violencia real y percibida) y la limitación a la libertad de expresión, lo que determina el uso de prácticas que dañan el quehacer social de la profesión periodística.
\end{abstract}

\section{Abstract}

The objective of this quantitative research is to describe the labor, economic, security and freedom outlook in which a group of journalists carry out their work in a context of high violence in Veracruz state, the most dangerous to practice this profession in Mexico. In addition, the work proposes the existence of a correlation between the fact of suffering violence and the construction of their perception, as well as between violence and economic situation and violence and freedom of expression. The results of this study demonstrate that the idea of violence in the environment of the journalists analyzed is related to the degree of direct exposure to violence (attacks, threats, assaults) to which they have been exposed, in addition to the fact that there is a positive correlation between exposure to violence (constructed by real and perceived violence) and the limitation of freedom of expression, which determines the use of practices that harm the social work of the journalistic profession.

\section{Palabras Clave}

Periodismo, Violencia, Precariedad económica, Libertad de expresión, Veracruz

Keywords

Journalism, Violence, Economic insecurity, Freedom of expression, Veracruz

${ }^{1}$ Universidad Iberoamericana

\section{Introducción: Veracruz, el estado más peligroso para los periodistas}

Han pasado más de 13 años desde que México incursionó en una nueva narrativa del poder denominada la «Guerra contra el Narcotráfico», conflicto declarado por el Estado que en sus primeros seis años dejó un saldo de $121 \mathrm{mil}$ asesinatos (Proceso, 30 de julio de 2013) y que también tendría relación con el incremento de delitos contra la libertad de expresión (Del Palacio, 2015), las agresiones contra periodistas (González, 2017, p.162), las complicaciones al ejercicio periodístico (Hernández, 2016) y la ampliación del término de «zona de conflicto» para incluir también la violencia generada por el crimen organizado como forma de 
presión a medios de comunicación y comunicadores (Sánchez, 2017, p.262).

A partir del establecimiento del operativo Veracruz Seguro, en 2011, la cobertura de hechos de violencia ligada al narcotráfico aumentó en los periódicos locales veracruzanos, incluso en medios considerados conservadores, como el Diario de Xalapa, los cuales no solían cubrir la nota roja (Del Palacio, 2014, p.83). En este contexto de periodismo regional se debe destacar que los medios locales reportean más violencia que los nacionales (Graber, 1978; Shoemaker Reese, 1996, en Rodelo, 2014, p.63).

Fue en el sexenio de Javier Duarte de Ochoa (2011-2016) cuando se agravó la situación de la libertad de expresión, ya que Veracruz se convirtió en el lugar más peligroso de México para ejercer el periodismo e incluso destacó a nivel internacional tras sumar 17 periodistas asesinados en ese sexenio (Santiago, 2019, p.33). Esa fue la dinámica para quienes ejercían el periodismo en un estado incrustado en un país donde "la libertad de expresión y el derecho a la información se han visto limitados. Matar periodistas es un mecanismo permanente en el país para silenciar a los reporteros" (Leos, 2017, p.8).

Según datos de Reporteros Sin Fronteras, hasta el 2015, Honduras, Guatemala, México y Colombia fueron los países "más mortíferos de América Latina para los periodistas" (Sánchez, 2017, p.262). Dos años más tarde, Reporteros Sin Fronteras publicó el informe "Veracruz: los periodistas frente al Estado de miedo" en el que, además del conteo de víctimas mortales, también daban a conocer que la entidad tenía el segundo lugar en agresiones contra la prensa a nivel nacional (RSF, 2017, p.6), entre los que identifica como principales agresores no tanto a la llamada delincuencia organizada, sino a las autoridades: "Cotidianamente debían lidiar con amenazas, intimidaciones, una estrecha vigilancia y detenciones arbitrarias, efectuadas la mayoría de las veces por la policía estatal y los servicios de seguridad pública" (p.9). Para el 2018, tras una revisión de un período de cinco años, México fue considerado el segundo país más peligroso para ejercer el periodismo por la UNESCO (2019, p.3), sólo detrás de Siria y por arriba de Afganistán. El informe más reciente de Artículo 19 (2020, p.19) mantiene la alerta sobre el país: México permanece como uno de los países más peligrosos para los periodistas, con un índice de impunidad de $99.13 \%$ en los crímenes cometidos contra comunicadores.

De acuerdo con Sánchez (2017, p.263),“en mayor o menor grado, todos los comunicadores que cubren temas relacionados con las mafias y la violencia criminal están expuestos a riesgos y tienen un común denominador: la vulnerabilidad". En ese sentido, se visualiza una impunidad en la relación con la violencia contra los periodistas y la cobertura del crimen además de que "la violencia ha frenado una libertad de expresión y una calidad periodísticas que de otra forma serían robustas y visibles por sí mismas" (Márquez, 2015)

Quienes ejercen el poder han aplicado diversas estrategias junto con los medios de comunicación para llevar un discurso sobre la violencia que sea conveniente a sus intereses, sin embargo, los grupos criminales también han aplicado formas de posicionamiento de la información: "Filtraban información a ciertos periodistas, enviaban fotografías y/o videos, monitoreaban constantemente la cobertura que recibían y analizaban cómo eran presentados por cada medio" (González, 2017, p. 167). Esta cobertura de la violencia relacionada con el crimen organizado trajo para los periodistas consecuencias en sus rutinas y prácticas profesionales, además de inestabilidad laboral y económica, aspectos que interesa explorar en esta investigación.

Sánchez (2017, p.280) también advierte que México comparte, junto a Colombia y Brasil, la característica de que los periodistas se enfrentan a coberturas de temas relacionados con la violencia en un contexto de condiciones laborales adversas, por lo que "las organizaciones especializadas de prensa alertan que comunicadores mal pagados pueden estar más propensos a caer en ilegalidades como el soborno o a establecer relaciones de interés con las fuentes" (p.280). Es por eso que "de forma genérica, los periodistas lamentan el monto de su salario. Aunque sean apasionados del periodismo" (Maldonado, 2018, p.2).

El ingreso mínimo de un reportero mexicano para el 2020, según la Secretaría del Trabajo y Previsión Social (STPS), se estima en 275 pesos con 90 centavos diarios. Sin embargo, además del bajo sueldo, se debe considerar la "ausencia de otras prestaciones socioeconómicas como vacaciones, aguinaldo, seguridad social, seguro de gastos mayores” (Hughes, Garcés, Márquez y Arroyave, 2016, p.6). 
En este sentido, en un análisis comparativo entre los salarios de periodistas mexicanos y colombianos, el porcentaje de reporteros mexicanos que ganaban el salario más bajo era 2.5 veces mayor que el de sus homólogos colombianos (p.16), mientras que entre los mexicanos, el grupo de mayor porcentaje en cuanto a los salarios más bajos, con un 51.2\%, fue el de los trabajadores de la prensa diaria (p.7).

Es en este contexto económico y de violencia (a nivel nacional y específicamente en el estado de Veracruz) en el que el grupo analizado ha desarrollado su ejercicio periodístico, por lo que se investigará si existe relación entre el ejercicio del periodismo en un entorno de violencia y su situación económica y de libertad de expresión.

La pregunta de investigación se centró en el siguiente planteamiento: ¿qué relación existe entre el desempeño del periodismo en un contexto de violencia con la condición económica y la libertad de expresión de un grupo de periodistas veracruzanos?

Adicionalmente, se plantearon las siguientes hipótesis:

H1: Que la violencia directa sufrida por los encuestados repercute en su construcción de la percepción de la violencia.

H2: Que el fenómeno de la violencia en estos periodistas tiene relación con su precariedad económica.

H3: Que el fenómeno de la violencia en estos periodistas tiene relación con el impacto al ejercicio de su libertad de expresión.

Por lo tanto, los objetivos de este trabajo son lograr la descripción, con base en la revisión de datos estadísticos, del panorama de seguridad y de las condiciones económicas en el que el grupo de periodistas veracruzanos realiza su trabajo periodístico, junto con el análisis para determinar si existe o no una asociación entre las variables de violencia y las relacionadas con la situación económica y el ejercicio libre periodístico de la muestra seleccionada.

Pese a que no se trata de una muestra probabilística, dado que se desconoce el número real de periodistas que trabajan en Veracruz por la falta de un padrón o registro de esta profesión en la entidad, la aportación de este estudio radica en que se trabajó con periodistas de tres distintas zonas del estado; además ofrece un enfoque distinto al de los estudios que se han realizado en Veracruz (mayormente cualitativos) al explorar la situación económica y de libertad de los periodistas en un contexto de violencia, así como también permite estudiar la posibilidad de una correlación entre estas variables.

\section{Aproximación teórica}

De acuerdo con lo expuesto durante la introducción, se habrá de abordar algunos conceptos clave para la interpretación de las variables estudiadas y, posteriormente, habrá de agregarse un breve repaso teórico que permitirá entender de mejor forma lo que argumentado en la discusión y conclusiones.

En primer lugar se discutirá sobre la libertad de expresión y la libertad de prensa, derechos individuales amparados en los artículos 6 y 74 de la Constitución Mexicana, sobre los cuales "el ambiente de violencia ha impedido el ejercicio de estos derechos" ((Arribas, 2016, p.60)). Para la Comisión Nacional de Derechos Humanos (s.f.) la libertad de expresión se entiende como aquella que permite "buscar, recibir y difundir informaciones e ideas, ya sea oralmente, por escrito, o a través de las nuevas tecnologías de la información, el cual no puede estar sujeto a censura previa sino a responsabilidades ulteriores expresamente fijadas por la ley". Adicionalmente, la Carta Democrática Interamericana destaca a la libertad de expresión y de prensa como "componente esencial de toda sociedad democrática y fundamento de su existencia" (Ramírez, 2008, p.47). Para Márquez, dentro de la cultura periodística pasiva que distingue al periodismo mexicano, además de la fuerte dependencia reporteril a las fuentes oficiales, la libertad de expresión y la crítica condicionada también se utilizan como "moneda de cambio" (2015, p.17) en una relación entre la prensa y el poder.

Otro concepto que importa definir es el de precariedad económica o situación económica, el cual está ligado a la posesión de uno o varios empleos que tienen poca estabilidad o un alto riesgo de que se pierda el trabajo; ofrecen un ingreso bajo o insuficiente; carecen de protección social así como de posibilidad para proteger sus derechos (Guadarrama, Hualde y López, 2012, p.217-218); y también se incluyen la falta de una relación laboral formal y de leyes protectoras, así como condiciones laborales inseguras (Organización Internacional del Trabajo, 2014 pp.10-11). Por lo tanto, la precariedad laboral "consiste en un aumento de la vulnerabilidad de los trabajadores a consecuencia de las relaciones que definen 
la continuidad y su trayectoria laboral" (Cano, 1998, en Martínez, Marroquín y Ríos, 2019, p.116), mientras que lo contrario a esta precariedad es contar con un trabajo que provea lo suficiente para "poder cubrir las necesidades básicas, acceso al seguro social, vacaciones, incapacidades, aguinaldos, etc” (Martínez, Marroquín y Ríos, 2019, p.115).

La precarización del trabajo periodístico en Veracruz se ha delineado por las características económicas del estado, que sentaron una dificultad para fundar empresas mediáticas grandes de modo que los medios de comunicación suelen tener una relación de dependencia con el poder político (Del Palacio, 2015, p.25). Dicha dependencia se fundamenta en una práctica generalizada durante la historia contemporánea del país en la que el Gobierno federal ha "visto a los medios como aliados, incluso empleados, y la prensa ha estado muy lejos de cumplir el papel de 'perro guardián' que tiene en los regímenes democráticos modernos" (Del Palacio, 2017, p.1237).

Hallin y Manclini "sostienen que la relación entre medios y gobierno está basada en el clientelismo político" (Del Palacio, 2017, p.1235). Tal relación clientelar entre medios y el poder gubernamental bajo el contexto específico de Veracruz (Del Palacio, 2015, p.21) constituyó una línea convergente con el "control gubernamental de la dinámica del campo electoral, a la nueva configuración del campo político" (Zavaleta, 2011, p.228).

Respecto a la violencia, la Organización Mundial de la Salud (s.f) la define como el uso a propósito de la fuerza física o el poder "ya sea contra uno mismo, otra persona o un grupo o comunidad, que cause o tenga muchas probabilidades de causar lesiones, muerte, daños psicológicos, trastornos de desarrollo o privaciones". Sin embargo, Hannah Arendt propone una perspectiva diferente para la dicotomía poderviolencia relacionando el poder con el consenso y el concepto gramsciano de hegemonía, de tal forma que el poder sería "una acción concertada y buscado por sí mismo para el ejercicio de las libertades públicas" (Kohn, 2009, p.66) y, bajo esta idea, la violencia no sería un instrumento para ejercer el poder, sino una consecuencia de la erosión del mismo en el que la violencia fungiría como sustituto (p.66).

En el contexto del discurso y la narrativa de la «Guerra contra el Narco» no se puede abordar un único tipo de violencia, sino que tiene que considerarse como una mezcla de diversos tipos. Según Žižek (2009, pp.10-24) es visible la violencia subjetiva (real) a través de los encabezados criminalizantes que dan cuenta de la cifra de asesinados y sobre los que quieren fundamentar la sensación de inseguridad, lo que oculta la violencia sistemática (estructural), que es la que viene del sistema político y económico basado en el capitalismo. De la misma forma en la que Žižek se refiere a un triunvirato de la violencia, Aspe señala que en la pirámide social de la violencia convergen la violencia directa, la violencia cultural y la violencia estructural (2016, pp.81).

La consecuencia radica en que la violencia subjetiva puede reproducirse por medio de la violencia simbólica, definida como: "todo poder que logra imponer significados e imponerlos como legítimos disimulando las relaciones de fuerza en las que se basa su fuerza, agrega su propia fuerza, es decir, una fuerza específicamente simbólica, a estas relaciones de fuerza" (Bourdieu y Passeron, 1981, p.44). Es decir, que esta violencia no viene de una forma obvia o física sino que lo hace bajo la forma de la imposición de la visión hegemónica de percibir, pensar y actuar. Por eso "esta forma de violencia es ejercida a través del consenso, donde la cultura opera como un dispositivo que encubre y enmascara relaciones de poder" (Oehmichen, 2010, p.4).

La relación entre periodismo y violencia se analizará desde dos puntos. Primero, la violencia como tema periodístico y social relevante, fundamentado en el fenómeno de la narratividad, que es "aquello que permite que ciertas narrativas sean más narrativas que otras" (Soltero, 2016, p.141). El interés del periodismo en los temas de la violencia se debe a lo que despierta entre la población, de modo que los hechos de violencia, su frecuencia y lo impactante "aseguraron que los medios les dedicaran una cantidad considerable de atención, y que de manera consecuente también lo hiciera una amplia parte de la sociedad" (p.137).

El body count o conteo de cuerpos es la forma más recurrente del periodismo mexicano para narrar la violencia (Reyna, 2018, p.94), en la que "se da prioridad a la cuantificación de cuerpos sin vida antes que a otros aspectos" (p.100). Bajo este esquema predomina el uso de las fuentes oficiales (p.109), pero sobre todo se evita profundizar y cuestionar las relaciones de poder establecidas (p.107).

Respecto a la falta de investigación, esta se puede revisar desde diferentes causas: por un lado, una diferencia entre 
teoría y práctica de la reinterpretación cultural de términos como la objetividad, herencia del periodismo norteamericano, que en México ha conducido al privilegio de "las fuentes oficiales y a su discurso, y que deviene en el llamado 'periodismo declarativo', que tiende a la superficialidad y oficialismo y no a la investigación, verificación o contextualización" (Márquez, 2012, p.97). Por otra parte, la falta de investigación respecto a las noticias sobre la violencia y el narcotráfico también se origina en el círculo de las condiciones laborales, como los bajos salarios (que deviene en la contratación de periodistas no profesionalizados), la fuerte carga de trabajo y otras prácticas internas (Del Palacio, 2014, p.100). También es resultado de las amenazas e intimidaciones, tanto de grupos criminales como de las instituciones, lo que conlleva a la autocensura (González, 2017, p.164).

El otro análisis teórico entre periodismo y violencia se fundamenta en las relaciones de poder. Se abordará el periodismo como un campo social, según la definición de «campo» en la teoría social de Bourdieu como: "una estructura de relaciones de fuerza entre agentes individuales o institucionales que luchan por hacerse del capital simbólico" (Bourdieu, 1997, p. 109 en Meza y Enríquez, 2018, p.50). Bajo esta dinámica, el reportero se plantea como un agente dominado (Meza y Enríquez, 2018, p.50) sobre el cual se ejerce la violencia simbólica, definida previamente. Bourdieu y Passeron también explicarían que para legitimar esta violencia simbólica es necesario que exista "un proceso de identificación con los portadores del significado" (Reguillo, 2012, p.45). Así, su posición y práctica se observa de manera relacional con otros individuos del mismo campo y el reportero asume un habitus, que es una forma de percibirse a sí mismo y a los demás miembros de su campo social (Meza y Enríquez, 2018, p.51). Estos habitus, sin embargo, "no son permanentes ni homogéneos, ya que, sus trayectorias derivan en habitus individuales o variantes estructurales" (Bourdieu, 2007, p.98, en Meza y Enríquez, 2018, p.52).

Dentro del proceso de recolección de información, los periodistas interactúan con fuentes informativas, que son tanto instituciones como personas "encargadas de proporcionar material informativo a las redacciones" (Zaragoza, 2002, p.155). No obstante, en algunos casos las fuentes poderosas establecen límites para informar sobre alguna noticia, como es el caso de las fuentes oficiales, gubernamentales o políticas (Schlesinger, 1992, p.281). La preferencia de los medios hacia estas fuentes conlleva a que las mismas definan los temas de la agenda mediática (p.285). De manera formal, la relación entre periodistas y clase política se podría establecer como el de uso de fuentes de información y contextualizador, aunque también se ejercen prácticas informales en las que los comunicadores utilizan a sus fuentes políticas como clientes, proveedores económicos o dadores de empleos (Mar, 2013, pp.106-123).

De esta manera se presentan no sólo los conceptos clave usados en esta investigación cuantitativa, sino que este breve análisis teórico dota las bases para poder interpretar los resultados obtenidos de estas pruebas.

\section{Revisión de la literatura}

La relación entre las condiciones del ejercicio profesional de periodistas que informan sobre el narcotráfico y la violencia, se ha estudiado en la región de Centroamérica, puesto que en estos países "la cobertura de la violencia criminal ha llegado entonces a ocupar un lugar privilegiado en la agenda informativa de los medios de comunicación" (Sánchez, 2017, p.260). La investigación se enfocó en la relación entre vulnerabilidad y cobertura de la violencia, para lo que aplicó un cuestionario a 33 periodistas de Honduras, Guatemala, El Salvador, Nicaragua, Costa Rica y Panamá, quienes fueron agredidos por hacer su trabajo en la cobertura del crimen organizado (p.267).

31 de 33 respondieron haber tenido que extremar precauciones y cambiar rutinas como: utilizar rutas alternas en su vida diaria, dar aviso de sus actividades a un contacto de confianza, no hacer pública alguna postura personal en temas que se consideran delicados, limitar su socialización y salidas a lugares de esparcimiento (p. 274). La investigadora contrastó que "para las organizaciones de prensa, cuanto más aislado esté el periodista que cubre temas de violencia criminal, mayor será su vulnerabilidad" (p.275), no obstante, el 40\% los periodistas que participaron en este estudio cuantitativo respondió que prefería trabajar en solitario (p.275). Otras medidas que han tomado estos periodistas frente a las coberturas de la violencia son no firmar sus notas o utilizar una autoría genérica como ocurre en México y Colombia (p.276). El trabajo también revela que la reducción de la cobertura 
del narcotráfico y la autocensura son medidas utilizadas por los periodistas en riesgo (p.277). Si bien la encuesta no abarcó a comunicadores mexicanos, las referencias a este país son recurrentes, pues la autora consideró que México, como Centroamérica, "tiene regiones donde ejercer el periodismo es muy riesgoso" (p.280).

A nivel nacional, Márquez y Hughes (2017) destacan que se han producido pocas investigaciones cuantitativas desde una perspectiva laboral, profesional y demográfica de los periodistas mexicanos, sin embargo, en medio de la escasez de investigaciones de ese tipo, fue posible detectar que desde los años ochenta ya se registraba que los comunicadores trabajaban en una situación precaria (p.94). Por eso mencionan que es con Worlds of Journalism como por primera vez se aplicó un estudio a una muestra representativa del sistema de medios mexicanos y toda su geografía, este ofreció la posibilidad de "reflejar la realidad local y los desafíos que enfrenta el periodismo en México" (p.96). Si bien aquí se ahonda en las condiciones laborales y el perfil profesional de los comunicadores, también se incluyeron preguntas diseñadas para detectar el impacto de los distintos tipos de violencia en el periodismo mexicano (p.101).

En cuanto a la violencia y el impacto en el periodismo regional, tanto Rodelo (2009, p.105), como Nicolás (2018, p.94), relacionan las afectaciones a la libertad de expresión con el incremento de la violencia en Culiacán, Sinaloa y Coahuila; Lemini (2015, p.177) se refiere a la transformación del ejercicio periodístico frente a la violencia, así como a los ataques dirigidos a periodistas e instalaciones de medios. Mención aparte merece el trabajo sobre Tamaulipas del periodista Javier Valdez (2016), puesto que aunque no se trata de una investigación académica, posee un valor testimonial importante ya que través de las voces de periodistas en activo documentó (casi un año antes de su asesinato) que los periódicos ya no informan sobre hechos de violencia debido a la presión del narcotráfico.

Dentro del periodismo regional mexicano, también hay investigaciones sobre los cambios de rutinas entre periodistas que cubren violencia, aunque desde una perspectiva más cualitativa que cuantitativa. Por ejemplo, en Monterrey, Nuevo León, en un estudio con periodistas de media carrera, que poseían educación universitaria y que cubrían la fuente policíaca, se encontró que los cambios en sus rutinas de trabajo estaban ligados con el incremento de los niveles de peligro al momento de cubrir las noticias, identificándose la autocensura como una forma de autoprotección, además de otras medidas como no salir a reportear solos (2016, Torres, Torres y Gutiérrez, pp. 1252-1262).

En el caso específico de Veracruz, el ejercicio periodístico cambió fuertemente a partir del desarrollo de un régimen autoritario en Veracruz con, Fidel Herrera (2004-2010), período en el que cuatro periodistas fueron asesinados. El trabajo de Andrade vincula la subsistencia del régimen electoral autoritario con la limitación del acceso a la información (2018, p.58) y el impacto social del crimen organizado, este último, como factor para obstaculizar "la construcción de un régimen democrático al intervenir en los procesos electorales estatales o municipales en mayor o menor medida" (Loza, 2016, en Andrade, 2018, p.75), mientras que los medios de comunicación contribuirían a este régimen ya sea porque reporteros y periodistas se volvieron objeto de censura, agresiones o asesinatos o por las relaciones económicas entre figuras políticas que financian medios de comunicación (Andrade, 2018, p.75).

Del Palacio (2015, p.33) expone que la violencia contra los comunicadores veracruzanos tiene un trasfondo en el que la precariedad laboral interviene y entre los que destacan: la falta de profesionalización, los bajos salarios, la carencia de servicio médico, de seguridad laboral o prestaciones, la falta de protocolos de seguridad ante la cobertura de la violencia y la necesidad de laborar en varios medios al mismo tiempo.

Si bien los periodistas veracruzanos ya se encontraban en una situación de precariedad económica, pues en 2012 se apuntaba que además de la informalidad de los contratos y la falta de prestaciones sociales "en la prensa de las distintas regiones del estado los sueldos de los reporteros llegan 'en el más optimista y genérico de los casos, a cinco mil pesos' (mensuales)" (Mar, 2013, p.124), la situación de la violencia repercutió fuertemente en las rutinas, coberturas, ejercicio de la libertad de expresión y hasta en la seguridad personal de los reporteros que pasaron de cubrir aspectos de la delincuencia común a tocar temas ligados al narcotráfico (Santiago, 2019, p.21). Mientras el "Estado tiene determinados deberes para favorecer la libertad de expresión y la protección de las personas que ejercen el periodismo, así como de los medios de comunicación" (Comisión Nacional de Derechos Humanos, 
2019, p.37), el régimen subnacional de Veracruz — pese a incluirse dentro de una nación democrática - no cumplió con tal obligación.

En la zona conurbada Veracruz-Boca del Río, Mar Vázquez (2016) estudió el trabajo de reporteros del periódico Notiver y determinó que sus rutinas y habitus periodísticos experimentaron cambios a partir del 2011, en marco del aumento de la violencia en el estado y contra los comunicadores, con la entidad a la cabeza entre las de mayores agresiones contra la prensa (pp. 34-35). En el mismo espacio geográfico, también se ha investigado sobre la relación entre la cobertura de la violencia con el cambio de las rutinas de los periodistas, alteraciones en el núcleo familiar y presiones que terminan en deserción laboral (Del palacio, 2015, p.325).

Bajo este panorama, se propone desarrollar el análisis de las respuestas del grupo de periodistas veracruzanos con el fin de identificar si existe la precariedad laboral a la que se refieren investigadores como Márquez y Hughes (2017) o Del Palacio (2015), así como la relación de la violencia con el ejercicio periodístico (Del Palacio, 2017) (Mar, 2016), pero desde una perspectiva cuantitativa.

\section{Metodología}

La presente investigación es un estudio cuantitativo del tipo descriptivo y correlacional. De acuerdo con Sampieri y Mendoza (2018, p.106) "los estudios descriptivos comúnmente son la base de las investigaciones correlacionales", al tiempo que "pretenden especificar las propiedades, características y perfiles de personas, grupos, comunidades, procesos, objetos o cualquier otro fenómeno que se someta a un análisis" (p.108) a través de la obtención y el reporte de datos para "caracterizar conceptos, fenómenos, variables o hechos en un contexto determinado" (p.108), mientras que los correlacionales tienen como fin "conocer la relación o grado de asociación que existe entre dos o más conceptos, categorías o variables en un contexto en particular" (p.109).

Para responder la pregunta de investigación, así como los planteamientos de las hipótesis propuestas, se diseñó y aplicó una encuesta a periodistas veracruzanos para conocer los datos relacionados con el ejercicio periodístico y las condiciones laborales para medir y describir a este grupo en un contexto de violencia $\mathrm{y}$, posteriormente, realizar las pruebas para determinar si hay dependencia entre las variables relacionadas con los conceptos de violencia, ejercicio periodístico y precariedad económica.

Debido a que no existe un registro nacional o estatal de las personas que ejercen el periodismo en Veracruz, dada la carencia de regulación sobre la actividad por tratarse del ejercicio de un derecho fundamental, tal situación impide conocer el universo de los periodistas veracruzanos, de modo que, por razones de economía de tiempo y recursos, se decidió trabajar con una muestra no probabilística por conveniencia que incluyera a sujetos de las tres regiones (norte, centro y sur) con las que la Comisión Estatal de Atención y Protección para los Periodistas (CEAPP, 2018) divide el estado de Veracruz para sus diagnósticos sobre libertad de expresión en la entidad.

En total, la muestra se compuso por 51 periodistas agrupados en las zonas norte, centro y sur de Veracruz. El número se determinó por el grado de participación voluntaria de los mismos periodistas, es decir, la disponibilidad que tuvieron para contestar una encuesta distribuida por medio de Google Forms a través de aplicaciones de mensajería instantánea.

Tabla 1.

Características demográficas del grupo analizado

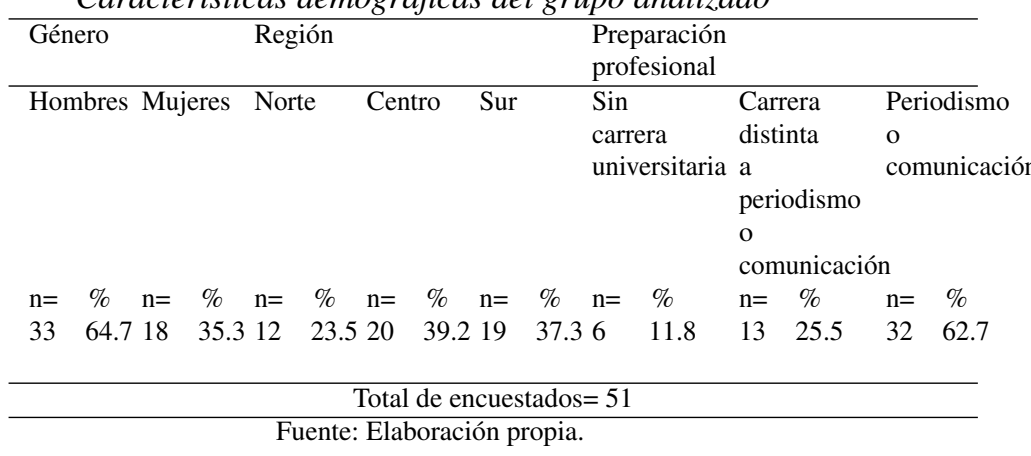

La recolección de datos se realizó a través de un instrumento formado por 43 preguntas agrupadas en cuatro aspectos principales:

a) Aspectos demográficos: estas preguntas tuvieron como fin conocer el género con el que se identifican, la región geográfica en la que trabajan y la preparación profesional que poseen.

b) Violencia: los cuestionamientos se relacionaron, a su vez, con dos situaciones: el primer grupo de preguntas tuvo como objetivo conocer si el periodista experimentó 
violencia directa, ya sea por estar en una situación de riesgo, sufrir acoso cibernético, ser objeto de amenazas, lesiones o enojo de funcionarios públicos, ser censurado, tener que cambiar sus rutinas, presenciar un delito, ser molestado por elementos de seguridad o trabajar en zonas consideradas inseguras. De estas preguntas se obtuvieron las variables observadas para conformar las dos variables latentes de "violencia experimentada directamente" y "percepción de la violencia", de las cuales se generó la variable latente de "violencia en los periodistas veracruzanos".

c) Condiciones laborales: en este apartado las preguntas se diseñaron para conocer el nivel de precariedad económica en el que se encuentran, de acuerdo con el número de trabajos que desempeñan simultáneamente, los tipos de medios para los que trabajan, si necesitan una fuente adicional de ingresos, si ganan menos del mínimo estipulado, si reciben su salario a tiempo, si cuentan con prestaciones, el nivel de estabilidad laboral, así como percepciones relacionadas con la situación económica de los medios y con su ejercicio profesional respecto a su ingreso. De estas variables observadas se construyó la variable latente de "situación económica".

d) Condiciones de libertad de expresión: las preguntas de esta última batería se enfocaron en aspectos relacionados a la autocensura, la pérdida de fuentes periodísticas y la limitación de investigación o escritura sobre diversos temas relacionados con el crimen y la violencia. Estos puntos se utilizaron para la conformación de la variable latente de "libertad de expresión".

En los resultados se incluyen las tablas con los datos de cada una de las variables latentes y las variables observadas que las componen, de acuerdo con esta división establecida, mismas que ilustran las frecuencias de los datos analizados.

La aplicación de la encuesta ocurrió entre el 14 de octubre y el 10 de noviembre de 2018 por medio de un enlace a Google Forms en donde se habilitó el formulario para responder y tras la fecha señalada quedó cerrado a participaciones. Posteriormente, los datos recolectados se trasladaron a una matriz para su análisis en el programa IBM SPSS Statistics (versión 26).

Para probar la hipótesis propuesta a raíz de la pregunta de investigación se utilizaron tres pruebas de correlación bivariada para determinar si existe correlación entre la variable de violencia con las de situación económica y libertad de expresión de la muestra y, en caso de resultar pertinente, el coeficiente de correlación de Pearson para conocer su dirección y magnitud.

\section{Resultados}

\subsection{Análisis descriptivo de las variables observables que componen los conceptos de violencia, situación económica y libertad de expresión}

La mayoría de los encuestados señaló trabajar en más de una empresa periodística. Para quienes trabajan en dos medios, la combinación de periódico impreso y digital fue la más elegida, mientras que para los que sólo tienen un trabajo, los medios digitales (con seis respuestas) casi duplicaron las menciones de los medios impresos (con 11). Además, el $62.7 \%$ de los periodistas tiene un trabajo adicional que no tiene que ver con los medios de comunicación.

En este panorama, más de la mitad de los entrevistados dijo ganar menos de 7 mil pesos al mes y no les pagan a tiempo, mientras que el $96.1 \%$ de los participantes consideró que su sueldo no es justo. También se observó que la mayoría manifestó preocupación por su situación económica, mientras que en el tema de la estabilidad laboral, se apreció un importante nivel de incertidumbre para los trabajadores.

En el caso de las prestaciones laborales, 26 (51\%) no cuentan con ellas y 24 (47.1\%) sí; no obstante, de los que sí tienen prestaciones, sólo 13 están afiliados al Instituto Mexicano del Seguro Social (IMSS) y cuentan con pensión y crédito para la vivienda; en cambio ocho respondieron que nada más tienen vacaciones y aguinaldo; uno dijo contar solamente con seguro médico y aguinaldo; uno nada más recibe aguinaldo; y otro nada más cuenta con seguro médico.

Además, 38 reporteros consideraron que la violencia ha afectado la economía de sus medios: las condiciones que señalaron fue que las ventas disminuyeron por la violencia, seguido de la menor contratación de publicidad y finalmente que la eliminación de la sección de nota roja impactó en los ingresos. 


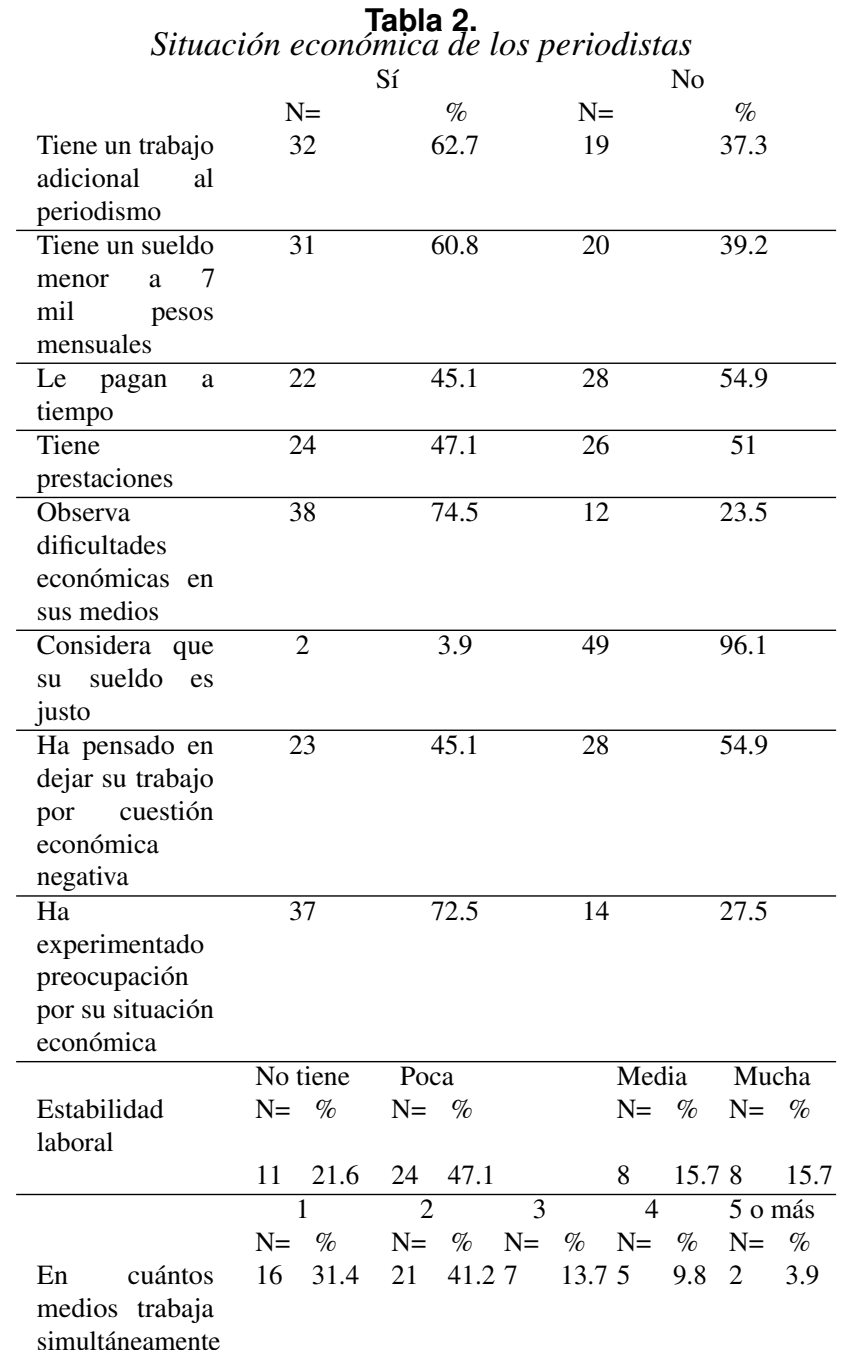

Fuente: Elaboración propia.

Respecto a la libertad de expresión, el $92.2 \%$ de los encuestados respondió que sí se ha autocensurado y los temas en los que más se han limitado están relacionados con la delincuencia: crimen organizado, narcotráfico, trasiego de combustible, secuestros, desapariciones y homicidios; mientras que los temas relacionados con el Estado, como corrupción y mal manejo de recursos, seguridad pública, ejército o marina, tuvieron una frecuencia mucho menor.

Poco menos de una cuarta parte de los encuestados señaló que dejó de escribir sobre un único tema, mientras que, por otra parte, la mayor parte de la autocensura abarca dos o más temas: ocho (15.7\%) respondieron que han dejado de escribir sobre dos y tres temas, respectivamente; cuatro (7.8\%) dejaron de hacerlo sobre cuatro temas; dos (3.9\%) lo hicieron en cinco temas; cuatro $(7.8 \%)$ en seis temas; tres $(5.9 \%)$ en ocho temas; y uno de los encuestados expuso que ha dejado de escribir sobre 12 temas distintos.

Tabla 3.

Libertad de expresión

\begin{tabular}{|c|c|c|c|c|}
\hline \multirow[b]{3}{*}{$\begin{array}{l}\text { Se ha limitado en el manejo de } \\
\text { temas periodísticos }\end{array}$} & \multicolumn{2}{|c|}{ Sí } & \multicolumn{2}{|c|}{ No } \\
\hline & $\mathbf{N}=$ & $\%$ & $\mathbf{N}=$ & $\%$ \\
\hline & 23 & 45.1 & 28 & 54.9 \\
\hline Se ha autocensurado alguna vez & 47 & 92.2 & 3 & 5.9 \\
\hline $\begin{array}{l}\text { Emplea recurrentemente la } \\
\text { autocensura }\end{array}$ & 24 & 47.1 & 27 & 52.9 \\
\hline $\begin{array}{l}\text { Ha experimentado pérdida de } \\
\text { fuentes }\end{array}$ & 29 & 56.9 & 19 & 37.3 \\
\hline $\begin{array}{l}\text { Ha dejado de escribir sobre } \\
\text { crimen organizado }\end{array}$ & 33 & 64.7 & 14 & 27.5 \\
\hline $\begin{array}{l}\text { Ha dejado de escribir sobre } \\
\text { narcotráfico }\end{array}$ & 25 & 49 & 22 & 43.1 \\
\hline $\begin{array}{l}\text { Ha dejado de escribir sobre } \\
\text { trasiego de combustible }\end{array}$ & 14 & 27.5 & 33 & 64.7 \\
\hline $\begin{array}{l}\text { Ha dejado de escribir sobre } \\
\text { secuestros }\end{array}$ & 13 & 25.5 & 34 & 66.7 \\
\hline $\begin{array}{l}\text { Ha dejado de escribir sobre } \\
\text { desapariciones }\end{array}$ & 12 & 23.5 & 35 & 68.6 \\
\hline $\begin{array}{l}\text { Ha dejado de escribir sobre } \\
\text { homicidios }\end{array}$ & 8 & 15.7 & 39 & 76.5 \\
\hline $\begin{array}{l}\text { Ha dejado de escribir sobre } \\
\text { corrupción y mal manejo de } \\
\text { recursos públicos }\end{array}$ & 8 & 15.7 & 39 & 76.5 \\
\hline $\begin{array}{l}\text { Ha dejado de escribir sobre } \\
\text { Seguridad Pública, Ejército o } \\
\text { Marina }\end{array}$ & 8 & 15.7 & 39 & 76.5 \\
\hline $\begin{array}{l}\text { Ha dejado de escribir sobre } \\
\text { fosas clandestinas }\end{array}$ & 7 & 13.7 & 40 & $\overline{78.4}$ \\
\hline $\begin{array}{l}\text { Ha dejado de escribir sobre trata } \\
\text { de personas }\end{array}$ & 3 & 5.9 & 44 & 86.3 \\
\hline $\begin{array}{l}\text { Ha dejado de escribir sobre } \\
\text { feminicidios }\end{array}$ & 2 & 3.9 & 45 & 88.2 \\
\hline $\begin{array}{l}\text { Ha dejado de escribir sobre } \\
\text { denuncias ciudadanas }\end{array}$ & 2 & 3.9 & 45 & 88.2 \\
\hline $\begin{array}{l}\text { Ha dejado de escribir sobre } \\
\text { asaltos y robos }\end{array}$ & 1 & 2 & 46 & 90.2 \\
\hline $\begin{array}{l}\text { Ha dejado de escribir sobre } \\
\text { conflictos sindicales }\end{array}$ & 1 & 2 & 46 & 90.2 \\
\hline $\begin{array}{l}\text { Ha dejado de escribir sobre } \\
\text { migración }\end{array}$ & 1 & 2 & 46 & 90.2 \\
\hline $\begin{array}{l}\text { Ha dejado de escribir sobre } \\
\text { Derechos Humanos }\end{array}$ & 0 & 0 & 47 & 92.1 \\
\hline
\end{tabular}
Fuente: Elaboración propia.

En relación a cuál es la principal problemática del periodismo veracruzano, siete $(13.7 \%)$ señalaron que es la violencia y las agresiones contra periodistas. En cuanto a las consideraciones de en qué ha cambiado el ejercicio periodístico, cuatro $(7.8 \%)$ mencionaron el hecho de que existe temor para realizar su trabajo, mientras que $23(45.1 \%)$ 
consideraron que hay más limitación en el manejo de temas y $24(47.1 \%)$, que recurren más a la autocensura.

En términos de percepción de la violencia en su trabajo, nueve de cada 10 participantes consideró su trabajo de periodista como peligroso, además de que más de la mitad expuso sentir peligro por la información que cubren, por el contexto de violencia en el estado o porque han sufrido amenazas; mientras que un número menor indicó tener miedo debido a los intereses económicos o políticos que pudieran llegar a afectar. El análisis reveló que menos de una tercera parte (20 encuestados) cree que hay más agresiones contra los periodistas, 18 consideraron que se ha vuelto más peligroso el ejercer dicha profesión y 39 participantes manifestaron que la violencia sí ha afectado el ejercicio del periodismo.

En cuanto a afectaciones anímicas de la violencia por su trabajo como periodista, más de la mitad (51\%) ha experimentado sentimientos relacionados con el miedo: ansiedad, ataques de pánico, temor a ser desaparecido o asesinado, pensamientos constantes sobre la muerte, intranquilidad o pesadillas; lo más recurrente fue la ansiedad, presente en ocho de cada 10 que han sentido miedo por su trabajo. Además, el 78.4\% externó miedo a ser asesinado o desaparecido.

Tabla 4.

Violencia percibida por los periodistas Sí No

\begin{tabular}{llllll} 
& \multicolumn{2}{c}{ Sí } & \multicolumn{3}{c}{ No } \\
& $\mathbf{N}=$ & $\%$ & $\mathbf{N}=$ & $\%$ \\
\hline $\begin{array}{l}\text { Considera que la violencia y } \\
\text { las agresiones contra periodistas } \\
\text { son el principal problema del } \\
\text { periodismo veracruzano }\end{array}$ & & & & & \\
\hline $\begin{array}{l}\text { Ha considerado cambiar de } \\
\text { profesión debido a la violencia }\end{array}$ & 7 & 13.7 & 44 & 86.3 \\
\hline Considera su trabajo peligroso & 30 & 58.8 & 18 & 35.3 \\
\hline $\begin{array}{l}\text { Tiene miedo de realizar su } \\
\text { trabajo }\end{array}$ & 4 & 7.8 & 47 & 92.2 \\
\hline $\begin{array}{l}\text { Considera que hay más } \\
\text { agresiones contra periodistas }\end{array}$ & 20 & 39.2 & 31 & 60.8 \\
\hline $\begin{array}{l}\text { Considera que se ha vuelto más } \\
\text { peligroso ser periodista }\end{array}$ & 18 & 35.3 & 33 & 64.7 \\
\hline $\begin{array}{l}\text { Ha experimentado miedo debido } \\
\text { a su trabajo }\end{array}$ & 26 & 51 & 25 & 49 \\
\hline $\begin{array}{l}\text { Ha tenido pensamientos sobre } \\
\text { ser asesinado o desaparecido }\end{array}$ & 40 & 78.4 & 10 & 19.6 \\
\hline $\begin{array}{l}\text { Considera que la violencia es la } \\
\text { principal afectación al ejercicio } \\
\text { periodístico }\end{array}$ & & & & & \\
\hline \multicolumn{1}{c}{ Fuente: Elaboración propia. } & & \\
\hline
\end{tabular}

Fuente: Elaboración propia.
Sobre la experiencia directa de la violencia, el $76.6 \%$ de los participantes ha estado alguna vez en situaciones calificadas de riesgo. Principalmente han recibido amenazas, seguido de ser objeto de agresiones físicas o el ser apuntados con un arma de fuego y, en menor proporción, malos tratos por parte de funcionarios, presenciar un hecho delictivo, ser censurados, ser intimidados por policías y ser difamados; cabe señalar que los periodistas pudieron mencionar más de un hecho de riesgo, dependiendo de qué situaciones han sufrido.

El contexto de violencia también reflejó que más de la mitad del grupo $(56.86 \%)$ ha tenido que cambiar alguna o varias de sus rutinas por cuestión de seguridad, mientras que el $43.1 \%$ no ha hecho cambios. Lo que más han cambiado son: las rutas hacia el trabajo o el hogar (13 menciones), el cambio de horarios y la implementación de protocolos de seguridad (nueve cada uno), cambio de hábitos (siete), cambio en coberturas informativas (seis) y reforzamiento de la seguridad en el domicilio (dos). Al igual que la pregunta anterior, los periodistas mencionaron más de un caso: 17 (33.3\% del total de encuestados) modificó un ámbito de sus rutinas; ocho (15.7\%) han cambiado dos ámbitos; tres (5.9\%) han cambiado en tres rubros; y sólo uno ha tenido cambios en cuatro aspectos. La violencia también ha significado para 29 de los encuestados (56.9\%) la pérdida de sus fuentes y la autocensura así como, para el mismo porcentaje, acoso cibernético y difamación después de la publicación de una nota periodística.

Tabla 5.

Violencia experimentada directamente por los periodistas

\begin{tabular}{|c|c|c|c|c|}
\hline & \multicolumn{2}{|c|}{ Sí } & \multicolumn{2}{|c|}{ No } \\
\hline & $\mathbf{N}=$ & $\%$ & $\mathbf{N}=$ & $\%$ \\
\hline Ha estado en una situación de riesgo & 39 & 76.5 & 12 & 23.5 \\
\hline $\begin{array}{l}\text { Ha cambiado sus rutinas por } \\
\text { seguridad }\end{array}$ & 29 & 56.9 & 22 & 43.1 \\
\hline $\begin{array}{l}\text { Ha experimentado acoso cibernético } \\
\text { o difamación en redes sociales }\end{array}$ & 29 & 56.9 & 20 & 39.2 \\
\hline Ha recibido amenazas & 29 & 56.9 & 21 & 41.2 \\
\hline Ha sido objeto de agresiones físicas & 14 & 27.5 & 36 & 70.6 \\
\hline Ha experimentado censura & 24 & 47.1 & 26 & 51 \\
\hline Ha presenciado un hecho delictivo & 17 & 33.3 & 33 & 64.7 \\
\hline $\begin{array}{l}\text { Ha sido blanco del enojo de } \\
\text { funcionarios públicos }\end{array}$ & 29 & 56.9 & 21 & 41.2 \\
\hline $\begin{array}{l}\text { Ha sido intimidado por agentes de } \\
\text { SSP }\end{array}$ & 5 & 9.8 & 45 & 88.2 \\
\hline $\begin{array}{l}\text { Trabaja en una zona de alta } \\
\text { inseguridad }\end{array}$ & 16 & 31.4 & 34 & 66.7 \\
\hline
\end{tabular}

Fuente: Elaboración propia. 
Finalmente, aunque para cerca de la mitad del grupo de profesionales veracruzanos $(51 \%)$, ser periodista otorga una satisfacción al generar un impacto positivo en la sociedad o contribuir a la resolución de problemas, un porcentaje ligeramente mayor $(54 \%)$ ha pensado en cambiar de profesión: entre ellos, se destaca que $21(41.2 \%)$ respondieron que es debido a condiciones económicas adversas, cinco $(9.8 \%)$ por la violencia y dos $(3.9 \%)$ por una combinación entre violencia y precariedad económica.

\subsection{Pruebas de correlación entre las variables latentes de violencia, situación económica y libertad de expresión}

Con base en las variables observadas relacionadas con violencia, situación económica y libertad de expresión, se construyeron otras tres variables latentes para medir de forma ascendente el nivel de exposición a la violencia, la precariedad económica y la afectación a la libertad de expresión. Estas variables numéricas se analizaron en el programa informático IBM SPSS (versión 26) por medio de la prueba de correlación bivariada para determinar si había correlación entre ellas, así como la dirección y magnitud de acuerdo con el valor del coeficiente de correlación de Pearson.

En total se realizaron tres pruebas de correlación bivariada: entre las variables de violencia experimentada directamente y de percepción de la violencia; entre las variables de violencia y de situación económica y entre las variables de violencia y de libertad de expresión.

En la primera prueba se determinó que existe una correlación $(\mathrm{P}<0.05)$ entre la violencia directa y la percepción de la violencia, además de que se trata de una correlación positiva moderada (.424), lo que indica que la percepción de la violencia aumenta en la medida en que lo hacen las exposiciones directas a la violencia.
Tabla 6.
Prueba de correlación entre variables latentes de violencia directa y percepción de la violencia

\begin{tabular}{ll}
$\begin{array}{l}\text { Violencia } \\
\text { directa }\end{array}$ & $\begin{array}{l}\text { Percepción } \\
\text { de } \\
\text { violencia }\end{array}$ \\
\hline 1 & 0.424 \\
& 0.002 \\
50 & 50 \\
\hline 0.424 & 1 \\
& \\
\hline 0.002 & \\
50 & 51 \\
\hline
\end{tabular}

Fuente: Elaboración propia.

En la segunda prueba el resultado indicó que no existe una correlación entre el nivel de violencia a la que se exponen los periodistas veracruzanos y el grado de precariedad económica $(\mathrm{P}>0.05)$.

Tabla 7.

Prueba de correlación entre variables latentes de violencia y precariedad económica.

Violencia Precariedad económica

\begin{tabular}{llll}
\hline Violencia & $\begin{array}{l}\text { Correlación } \\
\text { de Pearson }\end{array}$ & 1 & 0.152 \\
& & \\
& $\begin{array}{l}\text { Significancia } \\
\text { (dos muestras) }\end{array}$ & 0.335 \\
& $\mathrm{~N}$ & 50 & 42 \\
\hline $\begin{array}{l}\text { Precariedad } \\
\text { económica }\end{array}$ & $\begin{array}{l}\text { Correlación } \\
\text { de Pearson }\end{array}$ & 0.152 & 1 \\
\cline { 2 - 3 } & $\begin{array}{l}\text { Significancia } \\
\text { (dos muestras) }\end{array}$ & 0.335 & \\
& $\mathrm{~N}$ & 43 \\
\hline
\end{tabular}

En el último test se determinó que la violencia a la que se exponen los periodistas veracruzanos tiene relación $(\mathrm{P}<0.05)$ con el nivel de afectación de la libertad de expresión y se trata de una relación positiva media (.382), es decir, que en la medida que un periodista tiene un nivel más alto de exposición a la violencia, aumenta su nivel de afectación a la libertad de expresión, lo que significa una mayor autocensura, limitación en manejo de temas y riesgo de pérdida de fuentes. 
Prueba de correlación Tabla 8.

Prueba de correlación entre variables latentes de violencia y libertad de expresión.

Violencia

\begin{tabular}{llll}
\hline Violencia & $\begin{array}{l}\text { Correlación } \\
\text { de Pearson }\end{array}$ & 1 & expresión \\
\cline { 2 - 3 } & $\begin{array}{l}\text { Significancia } \\
\text { (dos muestras) }\end{array}$ & 0.382 \\
& $\mathrm{~N}$ & 50 & 0.006 \\
& & 50
\end{tabular}

\begin{tabular}{llll}
\hline $\begin{array}{l}\text { Libertad } \\
\text { expresión }\end{array}$ & $\begin{array}{l}\text { Correlación } \\
\text { de Pearson }\end{array}$ & 0.382 & 1 \\
\cline { 2 - 3 } & $\begin{array}{l}\text { Significancia } \\
\text { (dos muestras) }\end{array}$ & 0.006 & \\
& $\mathrm{~N}$ & 50 & 51 \\
\hline
\end{tabular}

\subsection{Discusión}

El resultado positivo de la prueba de correlación entre las variables de violencia directa y de percepción de la violencia $(\mathrm{P}<0.05)$, permite inferir que la percepción de la violencia para este grupo de periodistas veracruzanos se ve fuertemente influenciada por la experiencia directa del fenómeno. Puesto que las construcciones de la percepción de la violencia pueden exacerbar "el miedo de la población y favorecen esquemas de percepción y acción con los que se busca justificar el armamentismo, el militarismo y los estados totalitarios" (Oehmichen, 2010, p.3), debido a que los medios "juegan un papel central en la difusión de los discursos legitimadores de la violencia política" (Barreto, Borja, Serrano y López, 2009, p.742), existe la posibilidad de que la percepción de violencia de los periodistas se manifieste en la agenda pública.

Con base en los resultados, se entiende que los periodistas analizados no sólo han sido objeto de violencia directa, sino que también actúan como sujetos dominados al construir su percepción desde la manera en la que el poder hegemónico, en este caso, el régimen político electoral autoritario de Javier Duarte, lo ha dictado: "La narrativa popular es bastante clara en identificar al narcotráfico como el principal verdugo de periodistas mexicanos" (Ríos, 2013) como la mayor parte del problema de la inseguridad, narrativa que oculta la violencia sistemática y estructural, que viene del sistema político y económico basado en el capitalismo (Žižek, 2009, pp.10-24).

Por otra parte, la inexistencia de correlación entre la violencia a la que se exponen los periodistas veracruzanos y su condición económica $(\mathrm{P}>0.05)$, se puede explicar por el hecho de que la precariedad laboral es una constante en el periodismo veracruzano y nacional, incluso desde antes de la explosión de la violencia en la entidad. A pesar de trabajar en un contexto de violencia real y tener una alta percepción de la violencia en su entorno, en promedio, estos periodistas cuentan con 2.1 trabajos, la mayoría necesita ejercer una actividad económica adicional e independiente del periodismo, laboran con falta de prestaciones, los pagos se realizan a destiempo y cuentan con poca o nula estabilidad laboral. En un aspecto general, se considera que la situación económica del grupo es adversa desde que se observa que la mayoría cuenta con salarios por debajo del mínimo para un periodista, mismo que se sitúa alrededor de los 7 mil pesos al mes (Hughes, Garcés, Márquez y Arroyave, 2017, p.6).

Tales resultados coinciden con la precariedad detectada en otros estudios efectuados con periodistas a nivel nacional (Márquez y Hughes, 2017), en donde más de una tercera parte necesita tener empleos adicionales y la mayoría trabaja en más de dos medios simultáneamente (p.114), así como se ubica en un rango de salarios bajos y medios-bajos, (p.116 y 117). De esta manera, es posible detectar una congruencia entre la situación nacional del periodismo y la veracruzana, que no es una excepción en cuanto a precariedad laboral. Por lo tanto, aunque la violencia pueda agravar un poco esta condición, no es esta la que provoca tal situación precaria.

A pesar que la violencia ha incidido de forma negativa en el ejercicio periodístico, los comunicadores veracruzanos se sienten más vulnerados por las condiciones económicas precarias, como los bajos salarios o la falta de seguridad social, que por el riesgo en sí; así lo demuestra su percepción sobre la principal problemática del periodismo en el estado o las razones para cambiar de profesión. Itzia Miravete, Coordinadora de Documentación y Seguimiento de los Casos del Área de Protección y Defensa de la organización Artículo 19, ha señalado en específico sobre los reporteros veracruzanos que "sería un error ignorar que la falta de derechos laborales y sociales forma parte del problema estructural de las agresiones contra periodistas" (Santiago, 2019, p.96).

La correlación positiva entre el grado de violencia experimentada por los periodistas y el nivel de afectación en el ejercicio de su libertad de expresión $(\mathrm{P}<0.05)$ se debe entender desde el contexto específico de un periodismo regional en 
características distintas a las de los principios democráticos del país.

Aunque Veracruz se encuentra inscrito en una nación democrática, por lo que el "Estado tiene determinados deberes para favorecer la libertad de expresión y la protección de las personas que ejercen el periodismo, así como de los medios de comunicación" (CNDH, 2019, p.37), la entidad se observa como "una provincia autoritaria dentro de un país democrático, o bien en un régimen político electoralautoritario" (Del Palacio, 2017, p.1236), un “autoritarismo subnacional" (p.1236) inmerso en la democracia mexicana, lo que explica el porqué de la magnitud de las agresiones contra los periodistas; desde 17 asesinatos en un sexenio (Santiago, 2019, p.33) hasta el hecho de que, para quienes siguieron ejerciendo el periodismo en Veracruz en medio de la violencia, significara un cambio en sus rutinas, horarios, coberturas o temas de reporteo como en la implementación de mecanismos de seguridad o autoprotección (Del Palacio, 2017, p.323).

La falta de investigación periodística crítica respecto a la violencia tiene parte de su origen en las amenazas e intimidaciones, tanto de grupos criminales como, sobre todo, de las instituciones, lo que conlleva a la práctica de la autocensura (González, 2017, p.164). Para la investigadora Celia del Palacio "es posible afirmar que lo que ocurre en Veracruz constituye un ejemplo de lo que ocurre en otras entidades que se enfrentan a gobiernos autoritarios o poderosos grupos delincuenciales que han impuesto silencio" (2015, p.21). Como consecuencia, el miedo que lleva a la autocensura y la limitación de fuentes conduce al privilegio de "las fuentes oficiales y a su discurso, y que deviene en el llamado 'periodismo declarativo', que tiende a la superficialidad y oficialismo y no a la investigación, verificación o contextualización” (Del Palacio, 2017, p.323).

\section{Conclusiones}

Esta investigación cuantitativa permite abonar una perspectiva que complementa los estudios que ya han realizado investigadores sobre la relación de la violencia con el periodismo en Veracruz, desde un enfoque más cualitativo, lo que coadyuva a una mejor comprensión del fenómeno, especialmente al momento de poder establecer una relación entre dos aspectos.
Por medio de los resultados de este estudio cuantitativo pudo concluir que el grupo de periodistas veracruzanos, de tres regiones del estado, realiza su trabajo profesional en un contexto marcado fuertemente por la violencia, directa y percibida, y que esta situación de inseguridad está agudizada por la precariedad económica, no al revés.

El trabajo nos ha permitido deducir que el impacto de la violencia experimentada ayuda a prolongar la percepción de una situación de inseguridad para el periodista, por lo que a mayor exposición directa, se reforzará la percepción que tenga sobre su trabajo en relación con la violencia.

Resulta inquietante que los comunicadores se desempeñen con una constante preocupación en torno a su situación económica, incluso aún más que de su propia seguridad. Se considera que esta falta de condiciones mínimas para laborar deben tener un impacto en su desempeño periodístico, sin embargo, el estudio no es lo suficientemente amplio en este aspecto como para abordar ese tema, sin embargo, permite abrir la puerta a explorar esta línea de investigación que podría ofrecer un aporte relevante para el estudio del periodismo regional en Veracruz.

Aunque pudiese parecer obvia la hipótesis sobre la relación entre violencia y limitación a la libertad de expresión, la conclusión relevante aterrizada sobre este tema, es que tanto la coacción física (violencia directa) como el miedo (percepción de la violencia) en un contexto de violencia, agravan por medio de características como la autocensura, el desarrollo de un ejercicio periodístico incompleto que, de por sí, como lo han expuesto otros autores que citados en líneas anteriores, se destaca por la cobertura acrítica, la falta de investigación y la dependencia de fuentes oficiales por cuestiones de poder, económicas o por miedo.

Finalmente, se puede señalar que las respuestas obtenidas aquí también han permitido mirar hacia aspectos en los que no se logró profundizar, como la relevancia e impacto de la precariedad económica sobre la práctica periodística o la relación entre la violencia y el poder oficial, o la violencia y la construcción de las noticias, por mencionar algunos puntos. Sin embargo, estas mismas limitaciones enfocan la mirada a nuevas líneas de investigación, partiendo desde la base sólida que este trabajo permitió integrar. 


\section{Referencias}

Andrade, V. M. (2018). Violencia y régimen político en Veracruz, México: 1936-2016.. México.http://rcientific as.uninorte.edu.co/index.php/memorias/article/view/1 $0802 / 214421442924$

Arribas, A. (2016). Ser o no ser periodista en México. México.http://dspace.uhemisferios.edu.ec:8080/x mlui/bitstream/handle/123456789/535/arribas-INFO AMERICA.pdf?sequence $=1 \&$ isAllowed $=\mathrm{y}$

Artículo 19. (2020). Disonancia: voces en disputa. México.ht tps://disonancia.articulo19.org/wp-content/uploads/2 020/07/DISONANCIA-INF-A19-2019-PDF-WEB.p df

Aspe, A. (2016). Sobre violencia, cultura y reconciliación en México. México.http://www.scielo.org.mx/pdf/rfoi/v7n 12/2395-8936-rfoi-7-12-00077.pdf

Barreto, M., Borja, H., Serrano, Y., y López, W. (2009). La legitimación como proceso en la violencia política, medios de comunicación y construcción de culturas de paz. Universitas Psychologica, 8(3), 737-748.

Bourdieu, P. y Passeron, J. C. (1981). La reproducción. Elementos para una teoría del sistema de enseñanza. Barcelona: Editorial Laia

Comisión Nacional de Derechos Humanos. (2019). Informe especial sobre el contexto de violencia que enfrentan las personas que ejercen el periodismo y los medios de comunicación en el estado de Veracruz. México.https:// www.cndh.org.mx/sites/default/files/documentos/2019 -11/Violencia-periodismo-medios-comunicacion.pdf

Comisión Estatal de Atención y Protección para los Periodistas. (2018). Informe: Diagnóstico de la libertad de expresión de los Periodistas de Veracruz, junio $2018 .$. México.http://ceapp.org.mx/admin/pdf/2018-06-13/pd f/60.pdf

Comisión Nacional de Derechos Humanos. (s.f.). Derechos humanos. Libertad de Expresión. México.https://www. cndh.org.mx/derechos-humanos/libertad-de-expresion

Del Palacio, C. (2014). Labores de ritualización y mitificación en las secciones policiacas de tres periódicos de Veracruz a través de sus imágenes. México.http://www. scielo.org.mx/scielo.php?script=sci_arttext\&pid=S01 85-39292014000400004\&lng=es\&tlng=es

Del Palacio, C. (2015). Periodismo impreso, poderes y violencia en Veracruz 2010-2014. Estrategias de control de la información.. México.http://www.redalyc.org/arti culo.oa?id=34639625002

Del Palacio, C. (2015). Perspectivas éticas de la seguridad ciudadana en Chile y México. México.http://ri.uaemex. $\mathrm{mx} /$ bitstream/handle/20.500.11799/21446/Perspectiva s\%20éticas.pdf?sequence $=1 \#$ page $=318$
Del Palacio, C. (2017). Los medios de comunicación en un régimen electoral autoritario. El caso de Veracruz, México. México.https://www.researchgate.net/profile/ Andres_Rivarola_Puntigliano/publication/336578502 _Continentalism_and_Latin_American_geopolitical_t hinking/links/5da6ce1a4585159bc3d02131/Continent alism-and-Latin-American-geopolitical-thinking.pdf \#page $=1235$

González, R. A. (2017). Entre la espada y la pared: violencia y publicidad oficial como obstáculos para la modernización del periodismo mexicano. México.http: //www.redalyc.org/articulo.oa?id=59555067012

Guadarrama, R., Hualde, A., y López, S. (2012). Precariedad laboral y heterogeneidad ocupacional: una propuesta teórico-metodológica. México.http://www.scielo.org.m x/pdf/rms/v74n2/v74n2a2.pdf

Hernández, M. E. (2016). Periodismo regional en México y violencia del crimen organizado: un diálogo académico. . México.http://www.scielo.org.mx/scielo.php?script= sci_arttext\&pid=S0188-252X2016000300281\&lng=e s\&tlng=es

Hughes, S., Garcés, M., Márquez, M., y Arroyave, J. (2017). Rethinking professional autonomy: Autonomy to develop and to publish news in Mexico and Colombia. México.ht tps://www.researchgate.net/profile/Mireya_Marquez _Ramirez/publication/305384465_Rethinking_profess ional_autonomy_Autonomy_to_develop_and_to_publi sh_news_in_Mexico_and_Colombia/links/59f4d3f9ac a272607e2a87dc/Rethinking-professional-autonomyAutonomy-to-develop-and-to-publish-news-in-Mexic o-and-Colombia.pdf

Kohn, C. (2009). La dicotomía violencia-poder: una defensa de la propuesta arendtiana.. México.https://www.redaly c.org/pdf/1411/141115659004.pdf

Lemini, J. L. (2015). Violencia y periodismo regional en México. Ciudad de México, México: Juan Pablos Editor

Leos, S. E. (2017). Tratamiento informativo de los periódicos nacionales de México sobre los asesinatos de periodistas en el Estado de Veracruz (2011-2016). Universitat Autònoma de Barcelona. Tesis digital.

Maldonado, P. (2018). El salario de los periodistas, el ancla a su participación en las redes de clientelismo mediático. México.https://www.redalyc.org/jatsRepo/687/68758 478001/68758478001.pdf

Mar, G. H. (2013). Relaciones de poder entre periodistas y clase política: Los columnistas de la zona conurbada Veracruz-Boca del Río.México.https://cdigital.uv.mx/bit stream/handle/123456789/32581/marvazquezguadalup eh.pdf?sequence $=1 \&$ isAllowed $=\mathrm{y}$

Mar, G. H. (2016). Violencia y transformación del habitus periodístico en Veracruz: caso Notiver (2011-2013).México.http://balaju.uv.mx/index.php /balaju/article/view/2533/4424 
Márquez, M. (2012). Valores normativos y prácticas de reporteo en tensión:percepciones profesionales de periodistas en México.México.https://www.redalyc. org/articulo.oa?id=971/97124309010f

Márquez, M. (2015). Violencia y periodismo regional en México. Ciudad de México, México: Juan Pablos Editor

Márquez, M. y Hughes, S. (2017). Panorama de los perfiles demográficos, laborales y profesionales de los periodistas en México: Reporte de Investigación. México.https://www.redalyc.org/pdf/687/6875091700 5.pdf

Martínez, K. A., Marroquín, J., y Ríos, H. (2019). Precarización laboral y pobreza en México.

Meza, A. y Enríquez, J.A. (2018). Violencia simbólica en el campo periodístico de Sonora, México. México.https: //www.redalyc.org/articulo.oa?id=687/68758478004.

Nicolás, M. T. (2018). El peligro de ejercer periodismo en México: Análisis de la cobertura informativa del asesinato de Javier Valdez según el enfoque del peace journalism. México.http://trace.org.mx/index.php/trace /article/view/381/355

Organización Internacional del Trabajo. (2014). Educación obrera para el trabajo decente. México.https://www.wh o.int/topics/violence/es/

Proceso. (30 de julio de 2013). Más de 121 mil muertos, el saldo de la narcoguerra de Calderón: Inegi. México.http s://www.proceso.com.mx/348816/mas-de-121-mil-m uertos-el-saldo-de-la-narcoguerra-de-calderon-inegi

Ramírez, D. (2008). La libertad de expresión en México amenazada por las agresiones a periodistas y la concentración de medios. México.https://www.reda lyc.org/pdf/325/32515007.pdf

Reguillo, R. (2012). De las violencias: caligrafía y gramática del horror. México.http://www.scielo.org.mx/scielo.p hp? script=sci_arttext\&pid=S1607-050X20120003000 $03 \& \operatorname{lng}=\mathrm{es} \& \operatorname{tn} \mathrm{ln}=\mathrm{es}$

Reporteros Sin Frontera. (2017). Veracruz: los periodistas frente al Estado de miedo. México.https://rsf.org/sites/ default/files/rapport_veracruz_es-2.pdf

Reyna, V.H. (2018). Objetividad y conteo de cuerpos en el periodismo sonorense. México.http://www.scielo.org.m x/pdf/rmcps/v63n233/0185-1918-rmcps-63-233-93.pd $\mathrm{f}$

Ríos, V. (2013). ¿Quién mata a los periodistas?. México.https: //www.nexos.com.mx/?p=15419

Rodelo, F. (2009). Periodismo en entornos violentos: el caso de los periodistas de Culiacán, Sinaloa. México.http: //www.scielo.org.mx/scielo.php?script=sci_arttext\&p $\mathrm{id}=$ S0188-252X2009000200005\&lng=es\&tlng=es

Rodelo, F. (2014). EEl tráfico de drogas y la delincuencia organizada en los medios mexicanos. Una comparación de los temas de las noticias en medios de distinto alcance y sector. México.https://www.redalyc.org/pdf/687/6873 1036003.pdf
Sampieri, R. y Mendoza, C.P. (2018). Metodología de la investigación. Las rutas cuantitativas, cualitativa $y$ mixta. México: Mc Graw Hill Education.

Sánchez, M. I. (2017). Periodismo bajo fuego: la nueva guerra del crimen organizado en Centroamérica. México.https: //dx.doi.org/10.15517/aeca.v43i0.31558

Santiago, V. A. (2019). Guerracruz: rinconcito donde hacen su nido las hordas del mal. Ciudad de México, México: Penguin Random House.

Schlesinger, P. (1992). Repensando la sociología del periodismo: estrategias de las fuentes y límites del centralismo en los medios. México.https://www.redalyc. org/pdf/316/31641416.pdf

Secretaría del Trabajo y Previsión Social. (2020). Salarios mínimos 2020. México.https://www.gob.mx/cms/uploa ds/attachment/file/525061/Tabla_de_salarios_m_nmo s_vigentes_apartir_del_01_de_enero_de_2020.pdf

Soltero, G. (2016). Construcción de la violencia en México. México.http://www.redalyc.org/jatsRepo/267/2674825 2010/index.html

Torres, A. C., Torres, M. y Gutiérrez, A, E. (2016). Procesos de autocensura y subjetividad en el trabajo periodístico en contextos de violencia y narcotráfico en Nuevo León. En C. Mateos y F. J. Herrero (Coords.). Tenerife, España: Sociedad Latina de Comunicación Social.http://www.re vistalatinacs.org/15SLCS/2016_libro/059_Torres.pdf

United Nations, Educational, Scientific and Cultural Organization. (2019). Intensified attacks, new defences: developments in the fight to protect journalists and end impunity. México.https://unesdoc.unesco.org/ark: /48223/pf0000371343

Valdez, J. (2016). Narcoperiodismo. Ciudad de México, México: Penguin Random House.

Zaragoza, C. (2002). Periodismo en la convergencia tecnológica: el reportero multimedia del Distrito Federal. México.https://www.redalyc.org/pdf/421/ 42118509.pdf

Zavaleta, A. (2011). Sobre: Williams Cortez Montané, Violencia simbólica en el campo político de Veracruz. México.http://ulua.uv.mx/index.php/ulua/article/view/1 252/pdf_51

Žižek, S. (2009). Sobre la violencia. Seis reflexiones marginales. Buenos Aires: Paidós.

\section{Agradecimientos}

Un profundo agradecimiento a los periodistas veracruzanos que participaron respondiendo la encuesta sobre las condiciones en las que realizan su trabajo. También a la Universidad Iberoamericana de la Ciudad de México y a su programa de Maestría en Comunicación por ser el espacio en el que se desarrolló la presente investigación, así como al Consejo Nacional de Ciencia y Tecnología 
Análisis del impacto de la violencia en la situación económica y el ejercicio de la libertad de expresión en un grupo de periodistas veracruzanos

(Conacyt) por el apoyo a dicho posgrado de calidad, lo que ha permitido la realización de este estudio. 